\title{
Combining Logic and Natural Language Processing to Support Investment Management
}

\author{
Marjolein Deryck $^{1,2}$, Nuno Comenda ${ }^{3}$, Bart Coppens ${ }^{3}$, and Joost Vennekens ${ }^{1,2}$ \\ ${ }^{1}$ KU Leuven, Dept. Computer Science, Campus De Nayer \\ ${ }^{2}$ Leuven.AI - KU Leuven Institute for AI, Leuven, Belgium \\ ${ }^{3}$ Coppens and Partners Consulting \\ \{marjolein. deryck, joost. vennekens\}@kuleuven. be \\ \{nuno.comenda, bart.jan.coppens\}@coppens-and-partners.com
}

The Knowledge Base Paradigm (KBP) advocates a strict separation between declarative domain knowledge and logical inference tasks that can be applied to this knowledge to solve problems of interest [2]. In this paper we report the results from a case study in which we combine the principles of the KBP with a Natural Language (NL) interface. The case was executed at an international financial institution. As a part of its service, an investment banker offers clients advice on the financial products to buy or sell. The clients' preferences can be expressed in an investment profile, that determines which assets are eligible for a specific investment. The eligibility of a specific asset depends on a plethora of interacting rules and constraints. Previously, a bank operator translated the several requests into lengthy programs that contain a lot of enumerations, repetitions, and complex nesting of if-then clauses and exceptions that need to be followed in the right order. This makes each creation of an investment selection program a complex and time consuming task. Furthermore, the result is hard to validate, which entails a substantial operational risk.

Our application allows the eligibility of financial products to be defined by means of controlled natural language (CNL). Each sentence is constructed from a number of building blocks that are selected step by step to get to a complete sentence. The resulting highly structured NL sentence is automatically translated to first order logic (FOL). The application also contains a deep learning NLP module that accepts free-form English. It proposes three CNL statements that are most likely to present the English sentence. The user then selects the most correct sentence, makes adjustments if necessary, and validates the result.

When completed, the KB can be used by different inference methods to perform multiple tasks in the problem domain. We use the IDP system with its associated FOL-based language as underlying reasoning engine [1]. A KB consists of three parts: a vocabulary that contains the ontology of the domain, a theory that contains rules and constraints on the concepts in the vocabulary, and a structure, that delineates the domain of the concepts, and typically gives an interpretation for some of them. The information that is declaratively stated in the $\mathrm{KB}$, can be used for different purposes. The inference task of model expansion can be used to decide on the eligibility of a specific asset. Given a theory $T$ (that contains the rules of eligibility), and an interpretation $I_{p}$ for part of its vocabulary $V$, the model expansion inference computes interpretations $I_{t}$ for the entire 
$V$ such that $I_{p} \subset I_{t}$ and $I_{t} \models T$ [4]. In our application we typically possess all the information on the asset, such that only the values of Eligible and NotEligible need to be computed. The optimize inference is used to find a combination of eligible assets that can be acquired at minimal cost. To this end we create an additional term $m$ that represents this cost. Given a theory $T$, interpretation $I_{p}$ and term $m$, the optimize inference will look for a model expansion $I_{t}$ of $I_{p}$ that minimizes $m$ [1]. This is, it will select a combination of assets with the lowest associated cost that follows the eligibility rules and given $I_{p}$. The propagation inference computes a set of facts that are consequences of $T$ given $I_{p}$, i.e., that hold in all model expansions $I_{t}$ of $T$ with $I_{p} \subset I_{t}$ [1]. In the application, the propagation works interactively: as soon as a new rule is created, the impact on the eligibility is immediately shown by coloring the asset green (eligible) or red (not eligible). The explanation inference traces the propagated values back to the given values of the interpretation $I_{p}$ [3]. The application allows the user to click on a propagated value and see immediately which atoms steered the decision. The theory comparison task uses the model expansion inference to compare two profiles. Active investors will typically update their profile regularly. In this case an automated comparison of two versions of the profile is helpful to ensure that correct amendments have been made. With the model expansion inference the logical equivalence of two theories can be checked by merging two theories $T_{1}$ and $T_{2}$, and adding the constraint that an asset can only be eligible in one of both theories. If no model $I_{t}$ that satisfies $T_{3}$ is found, the two theories are equivalent.

Application development A prototype with a real-life example KB and the described inference tasks were showcased to the company in a prototype. Following this, the company has launched a project to further develop this prototype into a production application. The first technical release in production was done in February 2021 and a second release with improved workflow for signing the profiles between counterparties was released in June 2021. As of the second release, clients from large investment banks have access to a sandbox environment for training purposes. A full commercial roll-out will be done by September 2021. The target users for this commercial release are operations teams in the treasury back offices of large investment banks globally (target around 500 users across 150 organisations). The correctness of the knowledge base was insured by performing empirical tests with profile descriptions with up to 20 rules, and applied to portfolios of up to 300 assets with response times less than 3 seconds. These represent reasonable tranches for proper business use. Any larger portfolios can be tested off line with reporting being sent when processing has finished. Compared with the manual creation of a profile, the operational risk linked to the automation is almost non-existent thanks to the two-step procedure to turn natural language sentences via $\mathrm{CNL}$ automatically into an $\mathrm{FO}(.) \mathrm{KB}$. Once the $\mathrm{KB}$ is created, the application supports multiple services, such as the selection of eligible assets, optimisation of the associated costs and explanation of unexpected results. 


\section{Acknowledgements}

This research received funding from the Flemish Government under the "Onderzoeksprogramma Artificiële Intelligentie (AI) Vlaanderen" programme.

\section{References}

1. Broes De Cat, Bart Bogaerts, M Bruynooghe, G Janssens, and Marc Denecker. Predicate logic as a modeling language: The idp system. In Declarative Logic Programming: Theory, Systems, and Applications, pages 279-329. ACM Books, 2018.

2. Marc Denecker. Building a knowledge base system for an integration of logic programming and classical logic. volume 5366, pages 71-76. Springer, 2008.

3. Marjolein Deryck, Jo Devriendt, Simon Marynissen, and Joost Vennekens. Legislation in the knowledge base paradigm: interactive decision enactment for registration duties. pages 174-177. IEEE, 2019.

4. Johan Wittocx, Maarten Mariën, and Marc Denecker. The idp system: a model expansion system for an extension of classical logic. In Proceedings of the 2nd Workshop on Logic and Search, pages 153-165. ACCO; Leuven, 2008. 